\title{
Wake Effect on HAT Tidal Current Power Device Performance ${ }^{\dagger}$
}

\author{
Chul-Hee Jo $^{1}{ }^{*}$, Kang-Hee Lee ${ }^{1}$, Jun-Ho Lee ${ }^{1}$ and Cristian Nichita ${ }^{2}$ \\ ${ }^{1}$ Inha University, Incheon, 402-751, Korea \\ ${ }^{2}$ University of Le Havre, Le Havre, 76063, France
}

(Manuscript Received Jun 16, 2011; Revised July 22, 2011; Accepted August 7, 2011)

\begin{abstract}
The rotor that initially converts the flow energy into rotational energy is a very important component that affects the efficiency of the entire tidal current power system. Rotor performance is determined by various design variables. Power generation is strongly dependent on the incoming flow velocity and the size of the rotor. To extract a large quantity of power, a tidal current farm is necessary with a multi-arrangement of devices in the ocean. However, the interactions between devices also contribute significantly to the total power capacity. Therefore, rotor performance, considering the interaction problems, needs to be investigated to maximize the power generation in a limited available area. The downstream rotor efficiency is affected by the wake produced from the upstream rotor. This paper introduces the performance of a downstream rotor affected by wakes from an upstream rotor, demonstrating the interference affecting various gabs between devices.
\end{abstract}

Keywords: TCP (Tidal Current Power), Renewable energy, Interaction effect, CWC (Circulating Water Channel), CFD (Computational Fluid Dynamics)

\section{Introduction}

Tidal current power is now recognized as one of the clean power resources in Korea, where there are many strong current regions on the west and south coasts. Various researchers have studied tidal current power systems. For example in Japan, the Darrious type has been studied by Shiono et al. (1999). Walsum (1999) has introduced the current power system in Fundy, Canada, while Jo et al. (2008) published experimental results on the applications of tidal current power systems in a cooling water weir.

Due to the limited areas available with a strong current, the arrangement of the devices is very important to maximize the efficiency and economic viability of the power farm. Several papers have introduced the interaction effects of devices in the area. Maitre et al. (2007) have published a paper on

\footnotetext{
${ }^{*}$ Corresponding author. Tel.: +82-32-860-7342, Fax.: +82-32-864-5850. E-mail address: chjo@inha.ac.kr.

Copyright $@$ KSOE 2011.
}

the interaction problem. Bahaj et al. (2007) have studied the characteristics of the wake of a single turbine, and Jo et al. (2007) presented the interference rates of the axial, transverse and diagonal arrangements of rotors for various incoming current speeds. However, the actual interference effect can be determined by various factors, including rotor diameter, rotor type, current speed, associated structure and current direction. The numerical approach can generate various conditions for hydrodynamic aspects, showing pressure and velocity distributions, and interference effects for multiarrayed formations. The numerical tool is very useful but should be confirmed and verified with an experiment and field test to ensure confidence in the reliability of the results.

In this study, CFD (computational fluid dynamics) is used to analyze selected cases for axial interferences based on the experiment by Jo et al. (2010). The mesh is generated by the discontinuous and offset mesh merge methods. The CFD results are 
compared with the experiment and are further developed for various conditions.

\section{CWC Experiment}

\subsection{Rotor specification}

Table 1 shows the specification of the rotor. The rotor was fabricated of plastic with a onedirectional airfoil shape. The three blade rotor diameter was $0.5 \mathrm{~m}$.

Table 1. Specification of rotor

\begin{tabular}{|c|c|c}
\hline \hline & Description & Specification \\
\cline { 2 - 3 } & Notation & P-1D3B \\
& No. of blade & 3 \\
P-1D 3 B & Diameter(m) & 0.15 \\
\hline
\end{tabular}

To verify the performance of the rotor, various currents were generated and the rpm was measured as charted in Fig. 1.

\subsection{Interaction effect}

Three identical rotors were placed in a CWC to investigate the interaction effect in the axial direction. The rpm were measured with $1 \mathrm{D}$ and $1.5 \mathrm{D}$ axial gabs between rotors as shown in Fig. 2 .

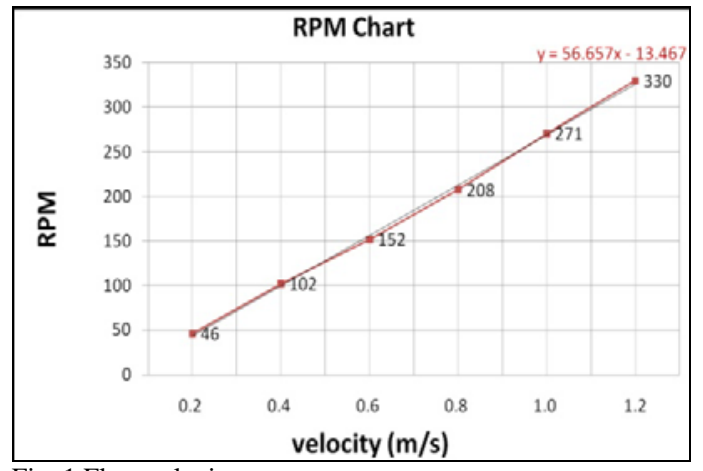

Fig. 1 Flow velocity vs. rotor rpm

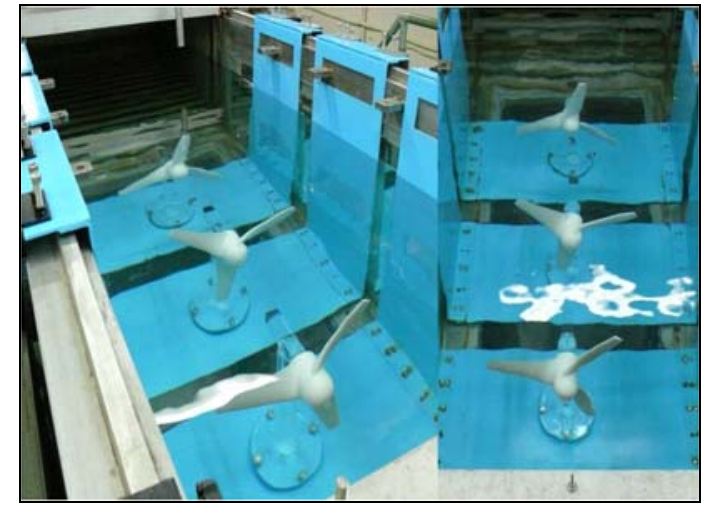

Fig. 2 Arrangement of rotors

The rpm deduction rates of downstream rotors were calculated and graphed considering the upstream rotor rpm.

As shown in Fig. 3, the rpm reduction rate between the $2^{\text {nd }}$ and $3^{\text {rd }}$ rotors was lower than that between the $1^{\text {st }}$ and $2^{\text {nd }}$ rotors. A similar result was observed for the $1.5 \mathrm{D}$ gab. It is expected that as the gab increases, the deduction rate will decrease as the flow pattern becomes more normalized.

\section{CFD analysis}

\subsection{CFD condition}

Static and dynamic simulations have been conducted for tidal current power devices that are located at specific distances. The static condition in this study means that the rotor is fixed at an incoming current and the dynamic condition refers to the rotating rotors at an incoming flow. Tables 2 to 4 show the analysis domain information together with the initial condition and meshing result.

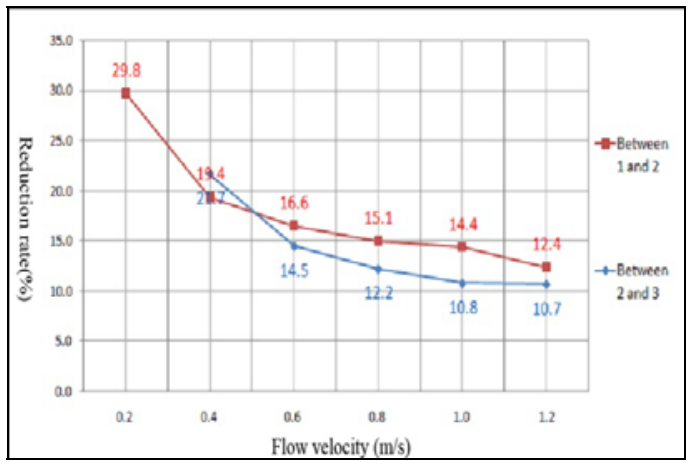

Fig. 3 RPM reduction rates between rotors at 1.5D 
Table 2. CFD domain specification

\begin{tabular}{c|c}
\hline \hline Description & Specification \\
\hline Length & $2.3 \mathrm{~m}$ \\
Breath & $2.6 \mathrm{~m}$ \\
Height & $0.9 \mathrm{~m}$ \\
\hline \hline
\end{tabular}

Table 3. Analysis condition

\begin{tabular}{c|c}
\hline \hline Description & Specification \\
\hline CFD code & ANSYS CFX 11.0 \\
Inlet condition & Normal speed(1.0 m/s) \\
Solid boundary & Stationary wall(no slip) \\
Outlet & Static pressure(1atm) \\
SST \\
Turbulent model & (Shear stress transport) \\
Interface condition & (General grid interface) \\
\hline \hline
\end{tabular}

Table 4. Mesh statistics

\begin{tabular}{c|r}
\hline \hline Generated mesh types & \multicolumn{1}{|c}{ Units } \\
\hline Total number of nodes & 657,177 \\
Total number of tetrahedra & $2,967,180$ \\
Total number of pyramids & 2,107 \\
Total number of prisms & 232,767 \\
Total number of elements & $3,202,054$ \\
\hline \hline
\end{tabular}

The ANSYS CFX v11 can automatically create meshes for three discrete areas; a general mesh for a circular channel domain, a detail mesh for a rotor area of dynamic rotation action and a very detailed mesh for the rotor tip as shown in Fig. 4. To prevent the discontinuity and mismatch along the boundaries and rotational rotors, the moving mesh and frozen-rotor interface options are applied. Fig 5 shows the modeled rotor. Boundary conditions and analysis information are shown in Table 3.

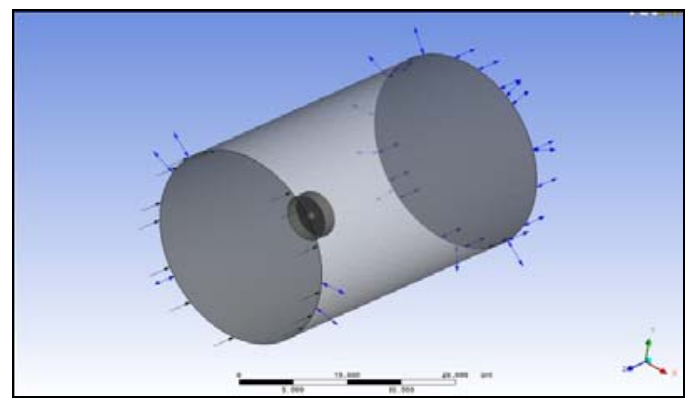

Fig. 4 Computational domain of turbine

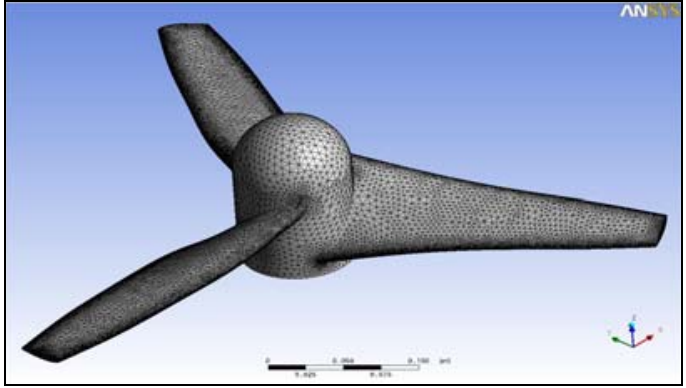

Fig. 5 Grid system of rotor

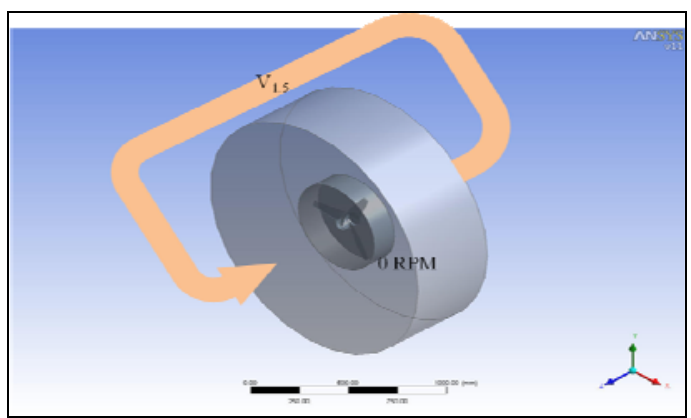

Fig. 6 Analysis procedure

\subsection{Interaction effects}

Due to the limited dimension of the CWC, the placement of a number of rotors is restricted. In CFD, several rotors were simulated to investigate the interaction effects between rotors with a periodic concept as shown in Fig. 6.

The analysis procedure is as follows:

(1) One rotor only is considered in CFD analysis and the incoming flow velocity to the $2^{\text {nd }}$ rotor is estimated. The flow velocity that can reach the $2^{\text {nd }}$ rotor RPM is calculated.

(2) The CFD model is modified considering the flow velocity measured from the experiment so that the incoming flow velocity to the $2^{\text {nd }}$ rotor in CFD becomes the same as that from the experiment.

(3) The $2^{\text {nd }}$ rotor is added in the modified CFD model and the incoming flow velocity to the $3^{\text {rd }}$ rotor is calculated.

(4) Using Fig. 1, the flow velocity for the rpm of the $3^{\text {rd }}$ rotor in Table 3 is estimated

(5) The CFD model is updated so that the calculated flow velocity is the same as that from the experimental measurement. 


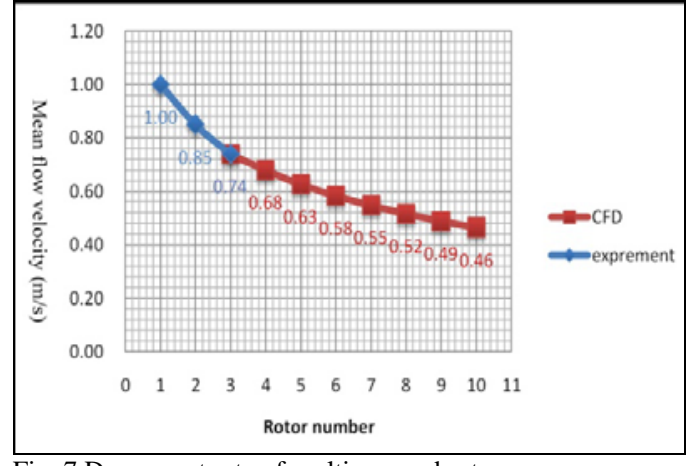

Fig. 7 Decrement rate of multi arrayed rotors

(6) By adding the $3^{\text {rd }}$ rotors in the modeling, the incoming velocity to the $4^{\text {th }}$ rotor is estimated numerically.

(7) The rpm equivalent to the calculated flow velocity is estimated in Fig. 1.

(8) Similar procedures are applied to analyze the multi-arrayed rotors. The axial gab between rotors is $1 \mathrm{D}$ with a $1 \mathrm{~m} / \mathrm{s}$ flow velocity. The 10 rotor arrangement was analyzed in CFD and the result is presented in Fig. 7.

The reduction rates for multi arrayed rotors are as shown in Fig. 7. The reduction rate decreases as the rotor is placed further behind, and it merges into the constant as the number of rotors increases.

\section{Conclusions}

The interference effects of tidal current power devices for an axial arrangement are investigated by CFD and an experiment for the selected cases.

From the study, it is observed that the fore rotor's interference is considerably more significant than the rear rotors. The study demonstrated that the power generation can be heavily affected by the interference of devices. Therefore, it is very important to optimize the device arrangement to maximize the power efficiency of the power farm.

The CFD method is helpful to understand the various aspects of multi-arrayed devices including interference effects. However, the CFD method should be verified together with the experiment since there are many assumptions and conditions applied in the program. It is found that the repeated shape can be generated efficiently with the offset mesh merge method.
A detail analysis for the specific environmental conditions considering rotor type and arrangement should be conducted to estimate the accurate power production rate considering the interference effects of the region.

\section{Acknowledgements}

This work was supported by the Human Resources Development of the Korea Institute of Energy Technology Evaluation and Planning(KETEP) grant funded by the Korea government Ministry of Knowledge Economy (No. 20094020100070) and by the Manpower Development Program for Marine Energy by the Ministry of Land, Transport and Maritime Affairs(MLTM).

\section{References}

[1] A.S. Bahaj, L.E. Myers, M.K. Thomson and N. Jorge, Characterising the Wake of Horizontal Axis Marine Current Turbines, Proceeding of $7^{\text {th }}$ European Wave and Tidal Energy Conference, Porto, (2007).

[2] C.H. Jo, K.H. Lee, Y.H. Rho and J.Y. Yim, Floating Tidal Current Power Application in Cooling Water Channel, The Korean Association of Ocean Science and Technology Societies Conference, Jeju, (2008) 2184-2187.

[3] C.H. Jo, K.K. Par and S.W. Im, Interaction of Multi Arrayed Current Power Generations, International Offshore and Polar Engineering Conference, Lisbon, (2007) 302-306.

[4] T. Maitre, S. Antheaume, C. Buvat, C. Corre and J.L. Achard, An Innovative Modelling Approach to Optimize the Design Configurations of Marine(river) Cross-flow Current Energy Converters' Farm, Proceeding of $7^{\text {th }}$ European Wave and Tidal Energy Conference, Porto, (2007).

[5] M. Shiono, K. Suzuki and S. Kiho, Experiments on the Characteristics of Darrieus Turbine for the Tidal Power Generation, Proceeding of the $9^{\text {th }}$ International Offshore and Polar Engineering Conference, Brest, Vol. 1 (1999) 123-128.

[6] W. Walsum, Offshore Engineering for Tidal Power, Proceeding of the $9^{\text {th }}$ International Offshore and Polar Engineering Conference, Brest, Vol. 1 (1999) 777 784. 